\title{
Linear viscoelastic fluid characterization of ultra-high-viscosity fluids for high-frequency damper design
}

\author{
with application to industrial high-precision motion stages
}

\author{
Cornelis A. M. Verbaan ${ }^{1} \cdot$ Gerrit W. M. Peters $^{2} \cdot$ Maarten Steinbuch $^{1}$
}

Received: 19 December 2014 / Revised: 4 June 2015 / Accepted: 19 June 2015 / Published online: 12 July 2015

(C) The Author(s) 2015. This article is published with open access at Springerlink.com

\begin{abstract}
This paper presents the use of a state of the art damper for high-precision motion stages as a sliding plate rheometer for measuring linear viscoelastic properties in the frequency range of $10 \mathrm{~Hz}-10 \mathrm{kHz}$. This device is relatively cheap and enables to obtain linear viscoelastic (LVE) fluid models for practical use in precision mechanics applications. This is an example of reversed engineering, i.e., turning a machine part into a material characterization device. Results are shown for a high-viscosity fluid. The first part of this paper describes the damper design that is based on a high-viscosity fluid. This design is flexure-based to minimize parasitic nonlinear forces such as hysteresis and stick-slip. In the second part of the paper, LVE fluid characterization by means of the damper setup is presented. Measurements are performed and model parameters are fitted by a non-convex optimization algorithm in order to obtain the frequency-dependent behavior of the fluid. The resulting fluid model is validated by comparison with a second measurement with a different damper geometry. This paper shows that LVE fluid characterization between $10 \mathrm{~Hz}$ and $10 \mathrm{kHz}$ for elastic high-viscosity fluids is possible with a motion stage damper for which the undamped behavior is known.
\end{abstract}

Keywords LVE fluid characterization · Damping ·

Ultra-high-viscosity fluid

Gerrit W. M. Peters

g.w.m.peters@tue.nl

1 Control Systems Technology Group, Department of Mechanical Engineering, Eindhoven University of Technology, Den Dolech 2, 5612, AZ Eindhoven, The Netherlands

2 Polymer Technology Group, Department of Mechanical Engineering, Eindhoven University of Technology, Den Dolech 2, 5612, AZ Eindhoven, The Netherlands

\section{Introduction}

A motion stage, as used for the illumination process of integrated circuits for instance, exists of a mechanical structure with force actuators to position the stage. This position is measured by sensors, which are connected to the feedback control system in order to stabilize the system and obtain the desired position. In addition, it guarantees performance in terms of position accuracy. The controller uses the difference between the actual position and the desired position - the position error - as input and transforms this information into an actuator force to minimize the position error as fast as possible. Such a controller acts as a frequency-dependent gain, which has to be designed as high as possible to prevent large position errors; the higher the controller gain, the stronger the motion stage reacts on a position error. From mechanical point of view, the motion stage can be assumed as a rigid body for low frequencies; the stage follows a set point or reference signal without showing internal deformation. At higher frequencies however, the stage structure shows flexible behavior (Gawronski 2004). This flexible behavior is a result of the limited stiffness of materials in combination with the mechanical design of the motion stage and results in specific resonance frequencies, also called natural frequencies, that correspond to deformation shapes, called mode shapes. Ultimately, the controller gain is limited by these natural frequencies and, therefore, the reaction speed of the motion system is restricted.

\section{Influence of damping}

Complex control approaches can be used to deal with this behavior and to increase the bandwidth as far as possible (Onishi et al. 1996; Balas and Doyle 1994), while retaining robustness (Bin 2010; Ho and Tomizuka 1996). In addition, optimization methods can be applied to improve the controller 
performance (Swevers et al. 2010). The extent to which the resonances limit the controller gain depends on (1) the structural design of the stage (Book 1993), (2) the position of actuators and sensors (Van de Wal and De Jager 2010), and (3) the damping present in the mechanical structure (Crandall 1970). In general, it can be stated that high natural frequencies and high modal damping values enable high controller gains. Current motion stage designs are designed to have high natural frequencies (Pedersen 2000) and, therefore, are based on materials (i.e., ceramics) with a high specific stiffness. These materials, however, usually contain low material damping. Stiffness and damping are sometimes combined to formulate optimization problems (Bodden and Junkins 1985). Another way to improve a systems' behavior is by increasing the damping of the mode shapes, which is called modal damping. A drawback of low modal damping is that vibrations in the stage, show large amplification factors and continue for a long time. This is detrimental to the transient response of the motion system. These observations lead to a continuous research effort to increase damping in mechanical designs (Soovere and Drake 1985; Johnson 1995). One method to add damping to a range of resonance frequencies is by adding separate dampers (Verbaan et al. 2013), which shows a method in which the damping of the resonant modes is increased by adding mechanical damping units to the motion stages' corners. These units consist of a mass, mounted on a parallel spring-damper configuration. The frequency range in which the damping has to be increased for the type of application aimed for is typically between 1 and $10 \mathrm{kHz}$. First of all, the damper behavior has to be linear in order to damp small as well as large amplitudes. This property is also beneficial during system analyses and design; nonlinear analyses are more difficult and time-consuming. In addition, most controller design strategies are based on linear theory (Skogestad and Poslethwaite, 2005). The next challenge is the small volume in which the damping mechanism has to fit. The third challenge is the relatively high damping value that has to be realized in that small volume. As solution to these problems, a fluid damper design based on an ultra-high-viscosity Newtonian fluid is proposed in (Verbaan et al. 2013).

\section{Linear viscoelastic fluid}

This solution is suitable to fulfill the three requirements, but it has to be taken into account that the properties of the damper, as function of frequency, might depend on the linear viscoelastic behavior of the applied fluid. Therefore, the linear viscoelastic behavior of the applied fluid has to be determined. Only linear viscoelastic (LVE) behavior is considered since the strains will be small. Different types of instruments are available to measure the LVE characteristics of a fluid, depending on the samples' viscosity and the frequency range of interest. In the low-frequency range (less than $10 \mathrm{~Hz}$ ), different types of dynamic viscometers are applied (Ferry 1969; Oakley and Giacomin 1994). In the high-frequency range (10-200 kHz), generally used for the investigating of microstructures and their interactions, a number of instruments based on quartz crystals and nickel tube resonators are available (Oosterbroek et al. 1980; Stokich et al. 1994). These instruments however measure fluid characteristics at discrete frequencies. Extended setups are available to obtain data at multiple discrete frequencies (Fritz et al. 2003; Schrag and Johnson 1971; Pechold 1959). The choice of instruments in the intermediate range is limited (Benzing and Russel 1981; Sittel et al. 1954; Konno et al. 1968). Although the frequency range of some instruments is close to the required range for this application, the range of applicable viscosities is often too low (Blom and Mellema 1984; Crassous et al. 2005).

The device as described in this paper combines a number of advantages for LVE characterization of ultra-high-viscosity fluids: (1) the frequency range that can be measured is relatively large and (2) the frequency range matches the frequency range of interest for practical applications, which avoids the necessity to change the temperature during the measurement. It enables to measure the LVE properties of ultra-highviscosity fluids $(>100 \mathrm{~Pa} \cdot \mathrm{s})$ in the frequency range between $10 \mathrm{~Hz}$ and $10 \mathrm{kHz}$, which (3) results in a relatively low production cost of this device $(\sim 2500)$.

The fluid used is a commercial synthetic hydrocarbon fluid (Rocol Kilopoise 0868 ) with a zero shear viscosity (ZSV) of $220 \mathrm{~Pa} \cdot \mathrm{s}$ and a density $\rho$ of $880 \mathrm{~kg} / \mathrm{m}^{3}$. No information about the viscoelastic properties is provided by the manufacturer.

\section{Outline}

A mechanical damper design for broad-banded damping based on the application of a high-viscosity fluid is presented in "Damper design," including its limits and assumptions. The dynamical model of the damper is described in "Damper dynamic model and mechanism characterization," as well as the characterization of the main mechanical part, which provides a point of departure for the LVE fluid measurements. "Linear viscoelastic damping" presents the linear viscoelastic theory of the Maxwell fluid model. "Results" presents measurements and discusses the linear viscoelastic fluid characterization approach. Parameters for the full damper model are determined by fitting the model on the experimental data. "Fluid model calculation and validation" represents the resulting LVE spectra, i.e., relaxation times and moduli, for different numbers of Maxwell modes. The best result is elaborated and the fluid model is shown. The model is validated by calculating the behavior of the damper setup with a different damping mechanism inserted and comparing the results with measured data. In addition, the fluid is measured with a different device and these results are compared with our findings. Conclusions and a discussion regarding the field of application and the 
elaboration of the damper setup are given in "Concluding remarks." Improvements regarding the damper design are addressed in order to improve the LVE model quality further.

\section{Damper design}

\section{Hardware configuration}

Figure 1a shows a photograph of the setup while the mechanical parts are shown in Fig. 1b, c. The damper assembly consists of a mass, mounted on a spring, and a damper in a parallel configuration. The mass can move in one direction over a relatively small stroke and is fixed in the other directions. The spring is a double-leaf spring guide. The ratio between the mass and the stiffness of the leaf springs determines the natural frequency of the damper mechanism. To test the damper, a force actuator is added, indicated by $F(t)$. The position of the mass $x(t)$ is measured optically. All these parts are indicated in Fig. 1a. The space between the leaf springs is used to locate the damping mechanism in. The fluid is applied in a sliding plate configuration to create a velocity-dependent force. It sits between two slot plates and a fin, positioned between the two plates, see Fig. 2. The fin moving relatively to the plates induces a shear flow. A flexible encapsulation is used to hold the fluid between fin and slot part. To study different damping values with the same fluid, two damper designs are used. Cross-sectional views of both damper mechanisms are given in Fig. 3. The left damper contains one fin, the right one two. In addition, the gap width is different in both cases. The dimensions are listed in Table 1. After assembling the slot and fin parts, the tolerances are less than $4 \times 10^{-6} \mathrm{~m}$ with respect to the gap width and less than $2 \times 10^{-6} \mathrm{~m}$ with respect to parallelism between the fin and the slot part.

To excite the damper mass, a voice coil (Akribis AVM245 ) is used which is driven by a current amplifier (custommade). The damper position is measured by a laser vibrometer (Polytech OFV-5000), and the data acquisition is done by a Siglab system that samples the laser vibrometer at $51.2 \mathrm{kHz}$
Fin part

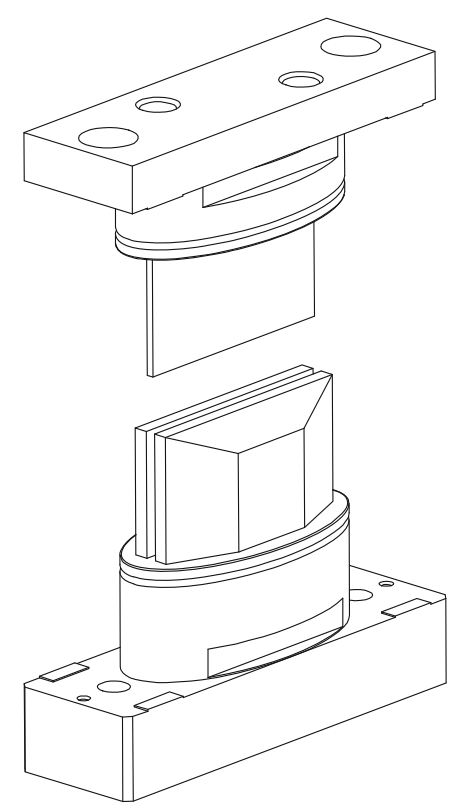

Fig. 2 Exploded view of the damper fin and slot parts

and provides also the signal for the amplifier. This input signal is also measured to calculate a frequency response.

\section{Limits and assumptions}

A sliding plate damper for high frequencies introduces side effects which can be divided into two groups: (1) geometryrelated effects and (2) frequency-dependent effects. A first geometrical effect is due to the finite length of the plates. From Table 1, it can be seen that the length-gap ratio amounts 133 for the single fin and 320 for the double fin. This ratio is so high that this effect can be neglected. A second geometrical effect is due to the difficulty to get the plates parallel to each other, especially with the normal forces acting on the moving fin. This design counteracts this problem in two ways; the damper part is symmetrical, which implies that the fin normal forces cancel each other. In addition, the double-leaf spring mechanism has a very high lateral stiffness, which prevents lateral displacements. A third geometrical effect is pumping of
Fig. 1 a Damper hardware with the functional components indicated. b Isometric view and c side view of the main mechanical parts: base, leaf springs, and mass (a)

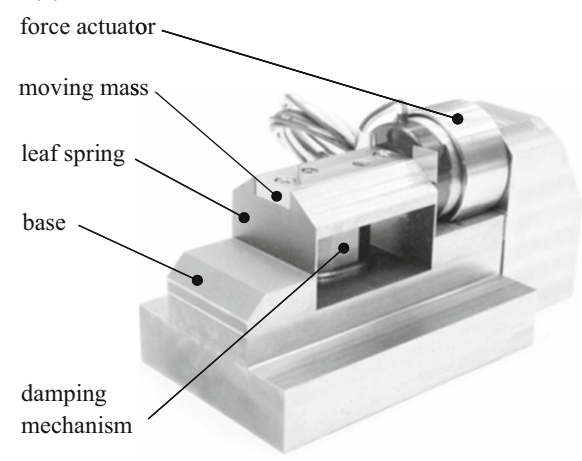

(b)

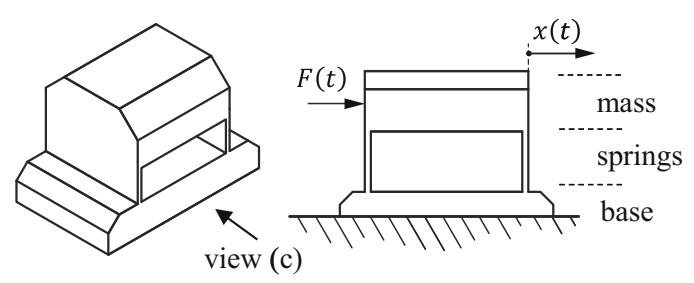

(c) 


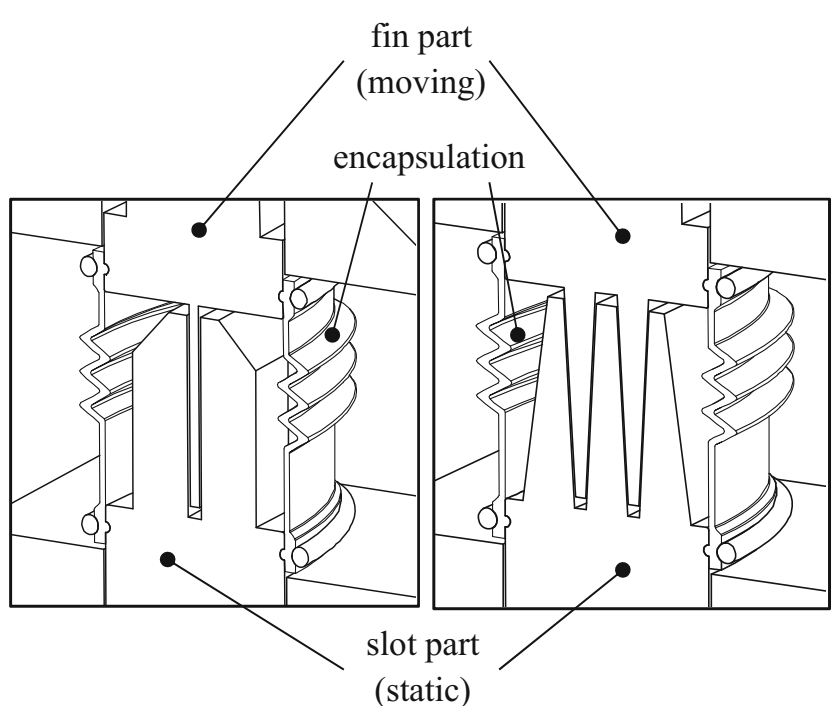

Fig. 3 Cross-sectional views of the two different damping mechanisms. Left, the single fin - as depicted in Fig. 2, right, the double fin

the fluid, which appears in the case of closed ends and introduces a flow opposite to the fin velocity, and therefore introduces a parasitic damping force. This problem is avoided by letting the gaps' ends open. The fin is shorter than the slot to have the same damping area over the full damper stroke. To avoid problems with the fluid running out, a flexible encapsulation is used which keeps the fluid inside. These effects all arise at low frequencies, at which the flow can be assumed homogeneous. The inertial effects determine to which frequency the flow can be assumed homogeneous.

A simple criterion for the time to establish homogenous, shear flow is given by (Schrag 1977).

$t_{c} \sim \frac{1}{\omega_{c}}=\frac{10 \rho h^{2}}{\eta}$

The maximum displacement amounts $10 \times 10^{-6} \mathrm{~m}$. In addition, measurements are performed with different input force amplitudes in order to validate the linear region of the fluid. The gap width $h$, as provided in Table 1, results in an estimated critical frequency of $250 \mathrm{kHz}$ for the largest gap width. This shows that a high fluid viscosity and small gap widths enable high frequencies without losing homogenous flow conditions.

Table 1 Parameters of both fin parts

\begin{tabular}{lll}
\hline & Dimension & Size $[\mathrm{mm}]$ \\
\hline Single fin & length $-l$ & 16 \\
& width $-w$ & 8.5 \\
& gap $-h$ & $120 \times 10^{-3}$ \\
Double fin & length $-l$ & 16 \\
& width— $w$ & 8.5 \\
& gap $-h$ & $50 \times 10^{-3}$ \\
\hline
\end{tabular}

\section{Damper dynamic model and mechanism characterization}

\section{Damper dynamic model}

Figure 4a shows a dynamic model that represents the behavior of the damper setup as shown in Fig. 1. Mass $m_{\mathrm{d}}$ represents the effective damper mass, mounted on stiffness $c_{\mathrm{d}}$ and damper $d_{\mathrm{d}}$. Mass $m_{\mathrm{m}}$ represents the mounting mass, which contains also the slot part and part of the connection of the moving mass. The spring with stiffness $c_{\mathrm{m}}$ and dashpot with damping $d_{\mathrm{m}}$ represents the connection to the fixed world or to a motion stage. The LVE damper behavior is included by an arbitrary number of Maxwell elements. The springs and dashpots in the LVE damper model generate forces as a result of the position and velocity differences between moving and mounting mass. Finally, in between the springs and dampers of the LVE damper model, additional degrees of freedom - with their corresponding masses $\left(m_{1} \ldots m_{n}\right)$-are added. These masses balance the force equilibria between the springs and the dashpots and can be considered as the mass of the fluid.

\section{Undamped characterization}

The dynamical behavior of the setup can be mainly characterized by two features:

- the mechanical design (mass and stiffness) and

- the high-viscosity fluid damper

In order to enable to calculate the behavior of the LVE fluid damper independently, the dynamic behavior of the mechanical part is characterized in advance. The corresponding model is shown in Fig. 4b and is a simplification of the model as shown in Fig. 4a. The natural frequency of the simplified model is determined by stiffness $c_{\mathrm{d}}$ and mass $m_{\mathrm{d}}$. Undamped measurements are performed with two different moving masses $m_{\mathrm{d}}$, i.e., with and without the fin part. This changes the natural frequency of the mechanism. The two corresponding frequency response functions are shown in Fig. 5.

In this figure, different frequency ranges are indicated:

a) low-frequency range, determined by the static stiffness of the leaf springs.

b) resonant behavior around the natural frequencies, clearly visible as peaks in the frequency responses.

c) higher frequencies behavior dominated by the moving mass.

d) at even higher frequencies, higher order dynamics is observed. 
Fig. 4 a Damper model with the LVE fluid behavior included. b Simplified damper model without the LVE fluid

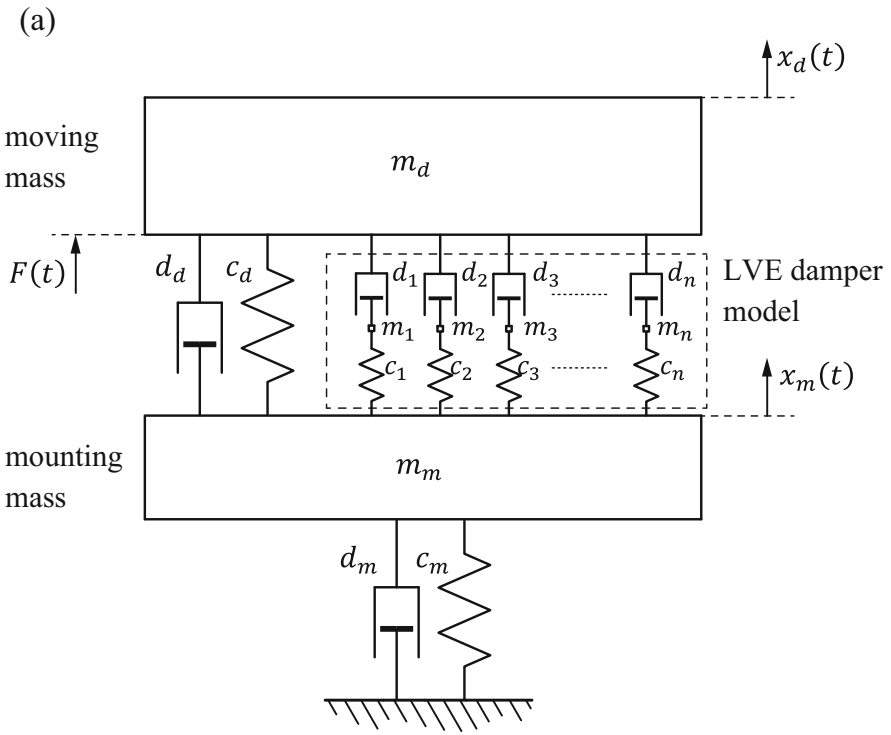

(b)

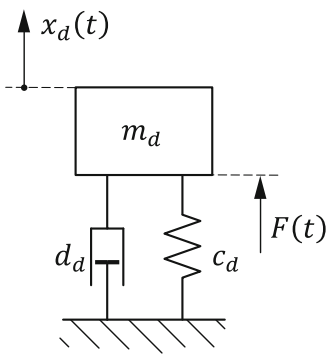

The value of the coherence of this measurement is close to 1 in the frequency region between $25 \mathrm{~Hz}$ and $15 \mathrm{kHz}$, which indicates that the measurement quality is rather good over this frequency range.

The two resonance frequencies, $f_{1}$ and $f_{2}$, can be determined with high precision. These two frequencies, combined with the mass difference between the two measurements $\delta m$, contain enough information to calculate the stiffness and mass of the undamped setup with:

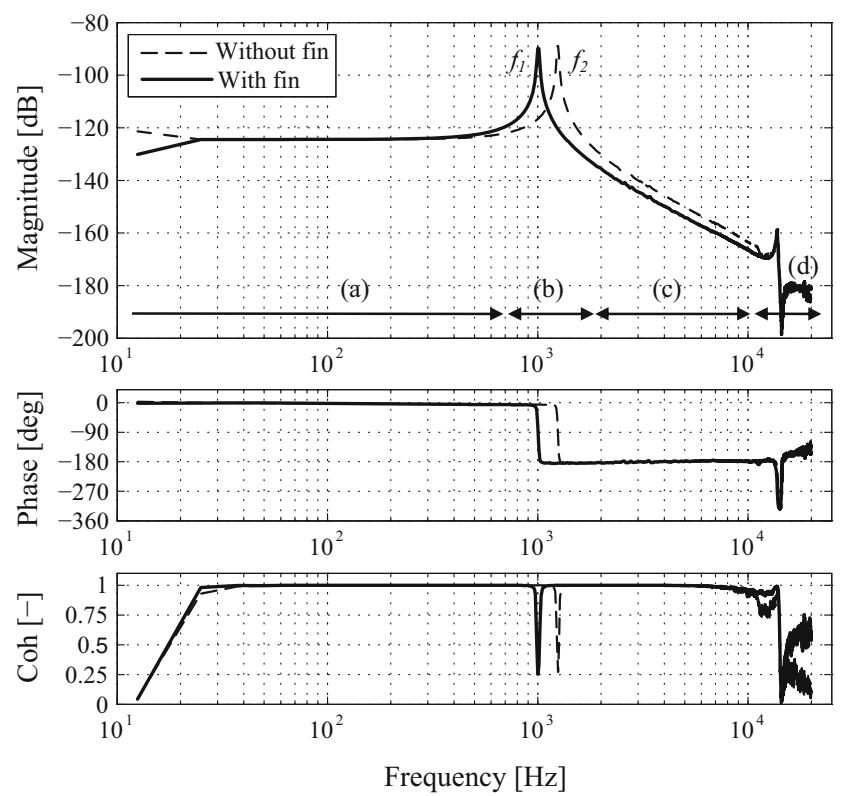

Fig. 5 Measured frequency responses of the undamped mechanism, with (solid line) and without fin (dashed line). Natural frequencies indicated by $f_{l}$ and $f_{2}$. Top: magnitude, middle: phase, lower: coherence (indicator for measurement quality). Ranges where specific system properties are dominant are indicated in the top figure; a stiffness, b resonance, c mass, and $\mathbf{d}$ high-order dynamics $\left[\begin{array}{cc}1 / \omega_{1}^{2} & -1 \\ 1 / \omega_{2}^{2} & -1\end{array}\right]\left[\begin{array}{c}k \\ m_{2}\end{array}\right]=\left[\begin{array}{c}\delta m \\ 0\end{array}\right]$

$m_{1}=m_{2}+\delta m$

Subsequently, the modal damping can be determined by calculating the amplification factor at the resonance frequency. This data is used in the subsequent steps as the model is extended. The modal damping can be estimated by the amplification factor at the resonance frequency, which is called the $\mathrm{Q}$ factor. This equation reads:

$Q=\frac{1}{2 \varsigma}$

The amplification in both cases amounts $36 \mathrm{~dB}$; this indicates that the modal damping of the systems amounts $0.79 \%$. This damping is transformed into a system parameter in Ns/m and included in the damper model as parameter $d_{\mathrm{d}}$. Table 2 represents the data as calculated from the measurements.

\section{Linear viscoelastic damping}

The linear viscoelastic (LVE) damping behavior of the fluid damper is represented by a multi-mode Maxwell model, see

Table 2 Parameter values of the undamped mechanism with the double-fin damper applied

\begin{tabular}{llll}
\hline & & Without fin & With fin \\
\hline Nat. freq & $\omega$ & $1212 \mathrm{~Hz}$ & $989 \mathrm{~Hz}$ \\
Stiffness & $c$ & $1,67 \mathrm{e} 6 \mathrm{~N} / \mathrm{m}$ & $1,67 \mathrm{e} 6 \mathrm{~N} / \mathrm{m}$ \\
Mass & $m$ & $28,8 \mathrm{~g}$ & $43,3 \mathrm{~g}$ \\
Damping & $\zeta$ & $0015-$ & $0015-$ \\
\hline
\end{tabular}


Fig. 4a, which gives a relation between stress, strain, and strain rate (Macosko 1994). The model requires material parameters: the elastic modulus $G_{0}[\mathrm{~Pa}]$ and the viscosity $\eta_{0}[\mathrm{~Pa}$. s]. The model as shown in Fig. 4a, however, describes displacements and velocities of moving masses, and the corresponding parameters are the system properties stiffness $c$ in $\mathrm{N} / \mathrm{m}$ and damping $d$ in $\mathrm{Ns} / \mathrm{m}$. Therefore, the Maxwell material parameters have to be transformed in system parameters before they can be used in the dynamic model. This relation can be found in the damper geometrical properties. In order to obtain the fluid material parameters, these geometrical properties should be excluded from the solution. For that purpose, firstly, the rheological expressions storage $\left(G^{\prime}\right)$ and loss modulus $\left(G^{\prime \prime}\right)$ have to be introduced.

The strain $\gamma$ and strain rate $\gamma$ in a fluid under harmonic excitation with frequency $\omega$ is described by

$\gamma=\gamma_{0} \sin (\omega \mathrm{t})$

$\dot{\gamma}=\omega \gamma_{0} \cos (\omega \mathrm{t})$

in which $\gamma_{0}$ is the strain amplitude. The shear stress is given by

$\tau=\tau_{0} \sin (\omega \mathrm{t}+\delta)$

in which $\delta$ is the phase lag of the stress w.r.t. the strain. This equation can be rewritten as

$\tau=\tau_{0}{ }^{\prime} \sin (\omega \mathrm{t})+\tau_{0}{ }^{\prime \prime} \cos (\omega \mathrm{t})$

Storage and loss moduli are defined by

$G^{\prime}=\frac{\tau_{0}{ }^{\prime}}{\gamma_{0}}$

$G^{\prime \prime}=\frac{\tau_{0}^{\prime \prime}}{\gamma_{0}}$

For the multi-mode Maxwell model, these moduli can be calculated as function of frequency using

$G^{\prime}(\omega)=\sum_{k=1}^{n} G_{k} \frac{\omega^{2} \lambda_{k}^{2}}{1+\omega^{2} \lambda_{k}^{2}}$

$G^{\prime}(\omega)=\sum_{k=1}^{n} G_{k} \frac{\omega \lambda_{k}}{1+\omega^{2} \lambda_{k}^{2}}$

in which $\lambda_{k}$ presents the characteristic times corresponding to the Maxwell modes. The magnitude and the phase are given by

$|G(\omega)|=\sqrt{G^{\prime}(\omega)^{2}+G^{\prime \prime}(\omega)^{2}}$

$\operatorname{angle}(G(\omega))=\tan ^{-1}\left(\frac{G^{\prime \prime}(\omega)}{G^{\prime}(\omega)}\right)$
The viscosity values $\eta_{i}$ corresponding to the Maxwell modes are given by

$\eta_{i}=G_{i} \lambda_{i}$

Figure 6 shows the geometrical parameters $(l, w, h)$ of the damping mechanism (see Table 1).

The relation between the stiffness and damping values in Fig. $4 \mathrm{a}$ and the fluid parameters modulus and dynamic viscosity are, respectively,

$c_{i}=\frac{2 l w}{h} G_{i}$

$d_{i}=\frac{2 l w}{h} \eta_{i}$

in which the factor 2 comes from the double-sided fin. Finally, the zero shear viscosity of the fluid can be calculated by

$\eta_{Z S}=\sum_{i=1}^{n} \eta_{i}$

i.e., the zero shear viscosity equals the sum of the mode viscosities.

\section{Results}

Figure 7 shows three measured frequency responses. The solid line represents the undamped behavior with the fin mass added, as presented in Fig. 5. The other two curves show results corresponding to the two different damping mechanisms (see Fig. 3). Stiffness $c_{\mathrm{m}}$ represents the coupling between the measurement table and the damper setup which is very high by mechanical design and, therefore, a relatively large value is implemented in the dynamic model. Subsequently, the frequency response of the Maxwell model is fitted to the measurement data. This results in system parameters, i.e., the stiffness and damping values, for every Maxwell model. To calculate the fluid behavior, model parameters have to be fitted on the measurement data and this is executed using an optimization algorithm. The complex response as function of the input frequency is measured and represented by the magnitude and the phase diagram. The (a)

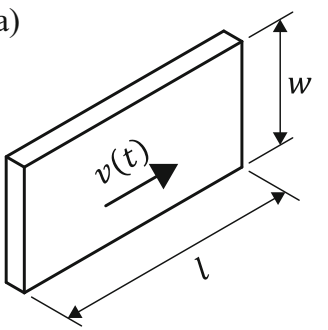

(b)

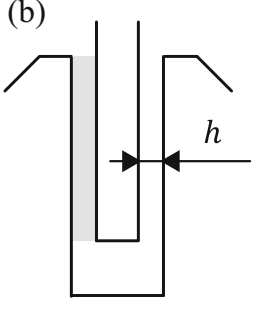

Fig. 6 a Fin and $\mathbf{b}$ gap and corresponding geometrical parameters 


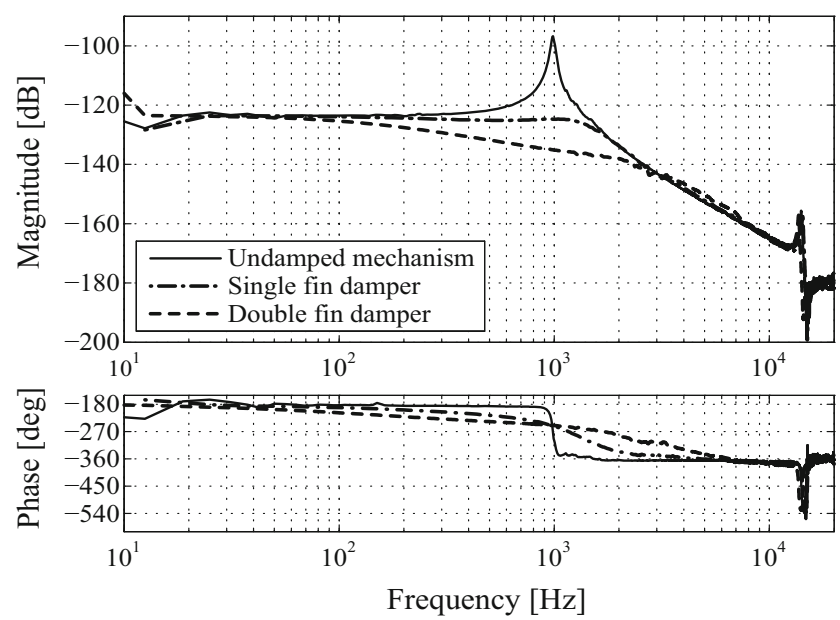

Fig. 7 Measured frequency responses for the undamped, the one-fin and the two-fin systems

magnitude information is used in the optimization algorithm to avoid time delay estimates in case of using the phase data.

The cost function is defined as

$\min _{x} J(x)$

in which parameter vector $x$ contains the parameters included in the LVE damping model

$x=\left[c_{1} \ldots c_{n} \mid d_{1} \ldots d_{n}\right]^{T}$

The parameter space is defined as

$l b \leq x \leq u b$

which defines the upper and lower bounds of the components of vector $x$. Further equality and inequality constraints are not included. In the definition above, the cost function is defined as

$J(x)=\max \left(a b s\left(\left\|R_{\text {meas }}\right\|^{2}-\left\|R_{\bmod }(x)\right\|^{2}\right)\right)$

(a)

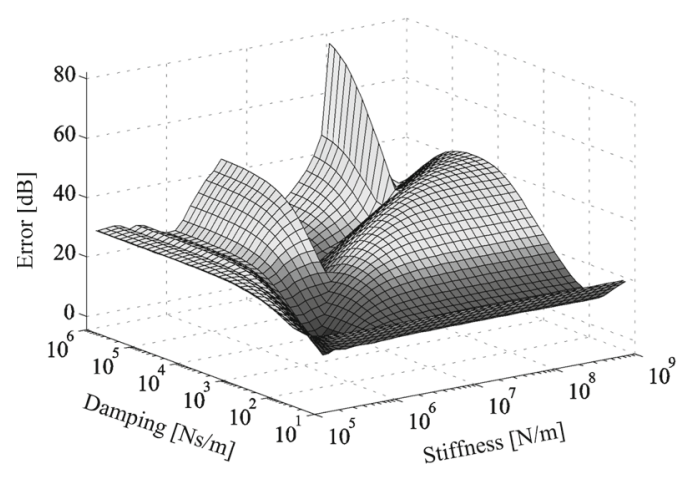

Fig. 8 a Error landscape between the measurement and the model for one Maxwell element. The $x$ - and $y$-axis represent the radix of the stiffness and damping values on logarithmic axes. The $\mathrm{z}$ direction shows the error between the measurement and the fit in decibels.
In this equation, $R_{\text {meas }}$ and $R_{\text {mod }}(x)$ describe the vectors with complex response data of respectively the measurement and the model as function of the frequency. The maximum value of the absolute error between the vectors with model data and measurement data is used for cost function evaluation. The stiffness and damping values are included for every single mode, which defines the length of parameter vector $x$ as two times the number of Maxwell modes. In this optimization criterion, all spring stiffness values in $\mathrm{N} / \mathrm{m}$ and damper values in $\mathrm{Ns} / \mathrm{m}$ can be chosen freely which implies that the time constants of the fluid modes are not determined on beforehand. Although the best fit for a fixed number of Maxwell modes is guaranteed by this approach, it does not necessarily result in an equally spaced distribution of the time constants of the modes. This optimization calculates $c_{1} \ldots c_{n}$ and $d_{1} \ldots d_{n}$ to minimize the magnitude difference between the dynamic model and the measurement. Masses $m_{1} \ldots m_{n}$ are determined beforehand and are not included in the optimization procedure. These amounts of mass are very low: it can be seen as the fluid mass itself and, due to the small gap width, amounts less than $0.1 \mathrm{~g}$. For calculation reasons, these masses should be chosen non-zero. Therefore, the masses are determined to be $10^{-2} \mathrm{~g}$, which enables a stable optimization procedure.

\section{Optimization approach}

Figure 8a shows the difference between the magnitude plots for a single Maxwell mode applied as LVE damper model. The horizontal axes are scaled logarithmically and show the damper values and the stiffness values, respectively. The vertical axis shows the maximum value of the error between the response magnitudes, see (22). Fig. 8 b shows a contour plot of the landscape of Fig. 8a, in which three gradient-based optimization paths are indicated with slightly different starting points. These optimizations prove that more local minima exist and that this problem has a non-convex character, even in

(b)

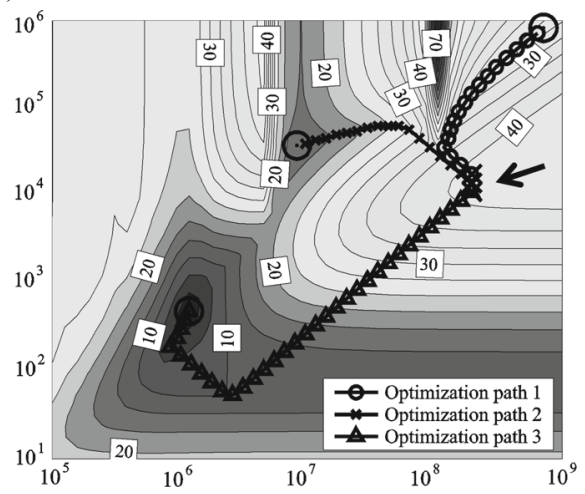

b Contour plot of the error landscape of Fig. 8a. Three optimizations are plotted in order to show the non-convex behavior within the optimization field. The three lines start at slightly different points (indicated by the arrow) and end in three different minima 
case of a single-mode Maxwell description. Therefore, nonconvexity is assumed in general for this optimization problem. In addition, discontinuous behavior is visible. These properties, non-convexity and discontinuity, require a non-convex optimization algorithm in order to try to find the global minimum.

A hybrid optimization procedure is applied, which starts with a genetic algorithm (GA) (Bäck 1996) to deal with the problem of multiple local minima. Although, in general, this type of algorithms do not guarantee that the global optimum is found, its efficiency can be influenced by choosing the correct algorithm parameters and initial population size (Diaz-Gomez and Hougen 2007). The solution of the GA is assumed to be in the vicinity of the global minimum and is used as starting point for a gradient-based optimization procedure (Rao and Rao, 2009). For this specific problem the parameter space is completely feasible, which is advantageous for the optimization speed, just as the absence of equality constraints.

\section{Fluid model calculation and validation}

\section{Parameter fit procedure}

Firstly, a single-mode Maxwell was fitted, see Fig. 9a. The maximum value of the error, after optimization, amounts $3.35 \mathrm{~dB}$. It appears that a part of the frequency range, between 2200 and $3400 \mathrm{~Hz}$, is excluded from the cost function due to parasitic dynamics in this frequency range. To decrease the error, a three-mode Maxwell model is fitted on the measurement data. The result is shown in Fig. 9b. The error has decreased to $0.40 \mathrm{~dB}$, a reduction of a factor 8 with respect to the one-mode Maxwell model. The error as function of the number of Maxwell elements is shown in Table 3, which shows that the error saturates for three Maxwell elements, i.e., more elements do not improve the solution further and show that a certain noise level is present in the measurement data.

(a)

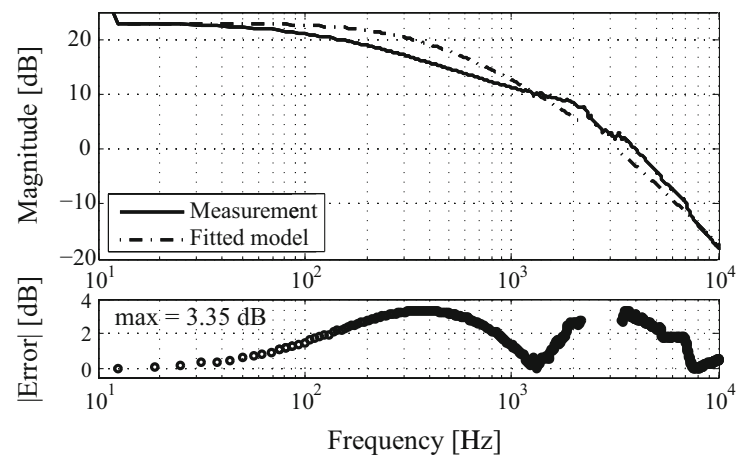

Fig. 9 a Measured results (solid line) and a single Maxwell mode fit (dash-dotted line) of the damped behavior with the double-fin setup. The upper plot shows the magnitude. The lower plot shows the error
Table 3 Convergence of the error as function of increasing number of Maxwell elements

\begin{tabular}{ll}
\hline \# Modes & Error $[\mathrm{dB}]$ \\
\hline 1 & 3.322 \\
2 & 0.938 \\
3 & 0.404 \\
4 & 0.414 \\
6 & 0.415 \\
7 & 0.416 \\
\hline
\end{tabular}

\section{Fluid parameters (three-mode model)}

The fluid parameters $G_{i}$ and $\eta_{i}$ can be calculated using the damping mechanism's geometrical parameters $l, w$, and $h$, see Eqs. (16) and (17). The time constants $\lambda_{i}$ of the different modes are calculated using (15). The resulting fluid parameters are listed in Table 4. The zero shear viscosity amounts 209.7 Pa's, see (18). This value underestimates the specified value of the manufacturer by $4.7 \%$. Figure 10 shows the storage and the loss moduli as function of the frequency. The compound behavior is represented by the solid lines, the separate modes by the dashed lines. The frequency range in which the measurement is taken with good quality is indicated in the figure. At low frequencies, the loss modulus is substantially higher than the storage modulus, which implies dominant viscous behavior.

\section{Model validation}

In the previous section, the double-fin measurement was used to derive a fluid model. In order to validate this fluid model, the reverse approach is applied; the resulting fluid model is used to predict the behavior of the damper with the single-fin mechanism, the result is compared with the corresponding measurement, see Fig 11. The magnitude (top) shows that the model provides a very good estimate of the measured behavior of the damper. The maximum error amounts

(b)

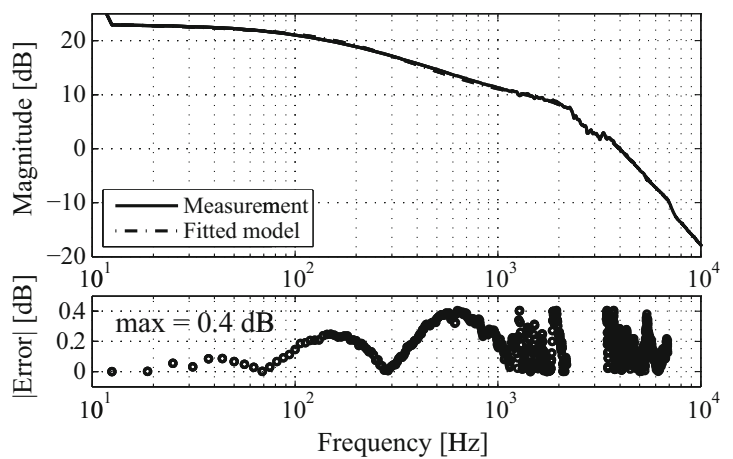

with the maximal error value of $3.35 \mathrm{~dB}$. b Three-mode Maxwell fit on the same measurement as in the left figure. The maximal absolute error amounts $0.40 \mathrm{~dB}$ 
Table 4 System parameter values and the corresponding fluid parameter values (three-mode model)

\begin{tabular}{|c|c|c|c|c|c|}
\hline & & M\#1 & $\mathrm{M} \# 2$ & $\mathrm{M} \# 3$ & \\
\hline C & $=$ & $1.51 \mathrm{e} 5$ & $4.29 \mathrm{e} 6$ & $5.72 \mathrm{e} 7$ & $\mathrm{~N} / \mathrm{m}$ \\
\hline$D$ & $=$ & 646.6 & 790.5 & 607.4 & $\mathrm{Ns} / \mathrm{m}$ \\
\hline \multicolumn{6}{|c|}{ Fluid parameters } \\
\hline$G$ & $=$ & 15,538 & $4.41 \mathrm{e} 5$ & $5.87 \mathrm{e} 6$ & $\mathrm{~Pa}$ \\
\hline$\eta$ & $=$ & 66.3 & 81.1 & 62.3 & $\mathrm{~Pa} \cdot \mathrm{s}$ \\
\hline \multicolumn{6}{|c|}{ Time constants } \\
\hline$\lambda$ & $=$ & $4.3 e^{-3}$ & $1.8 \mathrm{e}-4$ & $1.06 \mathrm{e}-5$ & $\mathrm{~s}$ \\
\hline
\end{tabular}

$1.7 \mathrm{~dB}$, see lower plot. This relatively small error indicates that the fluid model is good enough to predict the damper behavior over this frequency range.

\section{Single-fin fit and validation}

The fitting procedure is repeated on the single-fin measurements. The resulting fluid model parameter values are listed in Table 5. These values differ significantly from the values that are listed in Table 4 . The explanation of these differences can be found in the differences of the fin geometry: if the damping value is too low, the damping behavior cannot be extracted with enough accuracy. In this case, a single mode can describe the main characteristics of the damping behavior. The remaining two modes are difficult to place for the algorithm due to the low sensitivity of the displacement measurement. This phenomenon is elaborated upon in more detail in the discussion section in order to obtain an improved setup.
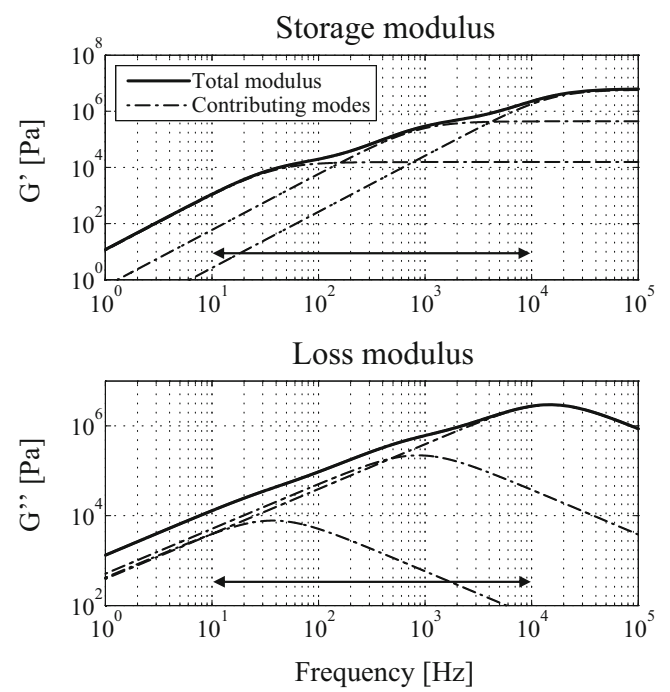

Fig. 10 Storage modulus $G^{\prime}(\omega)$ and loss modulus $G^{\prime \prime}(\omega)$ and the three separate modes. Arrows indicate the range of good quality measured results

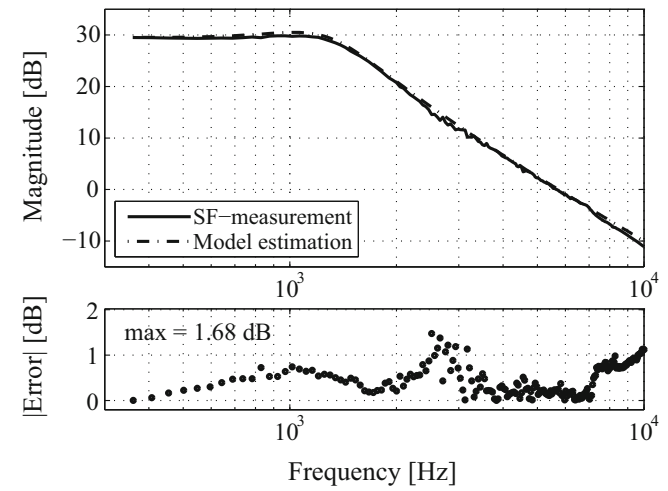

Fig. 11 Measured results with the single fin mechanism-continuous line - and the estimation by the 3-mode model-dash-dotted line. The lower plot shows the error between the prediction and the measurement

The zero shear viscosity in this case amounts $140.4 \mathrm{~Pa} \cdot \mathrm{s}$, which is $36 \%$ lower than specified. This model is validated by calculating the behavior of the double-fin setup and compared with the measurement of the double fin. In this case, the maximal error between the predicted behavior and the measurement amounts $2.3 \mathrm{~dB}$ which is larger than the error shown in Fig. 11.

\section{Fluid model validation by time-temperature-superposition}

To validate the quality of the obtained fluid model further, and thereby the quality of the setup, measurements are performed by means of time-temperature superposition (TTS) method. This method enables fluid characterization over very large frequency ranges (Olsen et al. 2001). The setup used is a Rheometrics ARES LS rheometer equipped with parallel plates. An isothermal frequency sweep from 215 to $0.1 \mathrm{rad} / \mathrm{s}$ with three logarithmically spaced points per decade was done. The maximum dynamic strain did not exceed $4 \%$ to ensure behavior in the linear viscoelastic region. Figure 12 shows the behavior of the fluid model as described in this paper and the curves measured by the TTS method for a reference temperature of $20{ }^{\circ} \mathrm{C}$. The fluid model, obtained by the setup as described in this paper, provides a relatively good fluid model up to at least $7 \mathrm{kHz}$, which was used in the fitting procedure. The waviness of the storage modulus in Fig. 12 is due to the fact that only three modes are applied to describe the fluid behavior over this frequency range.

Table 5 Resulting fluid model values for the single fin measurement

\begin{tabular}{llllll}
\hline & & M\#1 & M\#2 & M\#3 & \\
\hline$G$ & $=$ & $3.49 \mathrm{e} 5$ & 30.35 & $2.72 \mathrm{e} 9$ & $\mathrm{~Pa}$ \\
$\eta$ & $=$ & 69.5 & $5 \mathrm{e}-4$ & 70.9 & $\mathrm{~Pa} \cdot \mathrm{s}$ \\
\hline
\end{tabular}




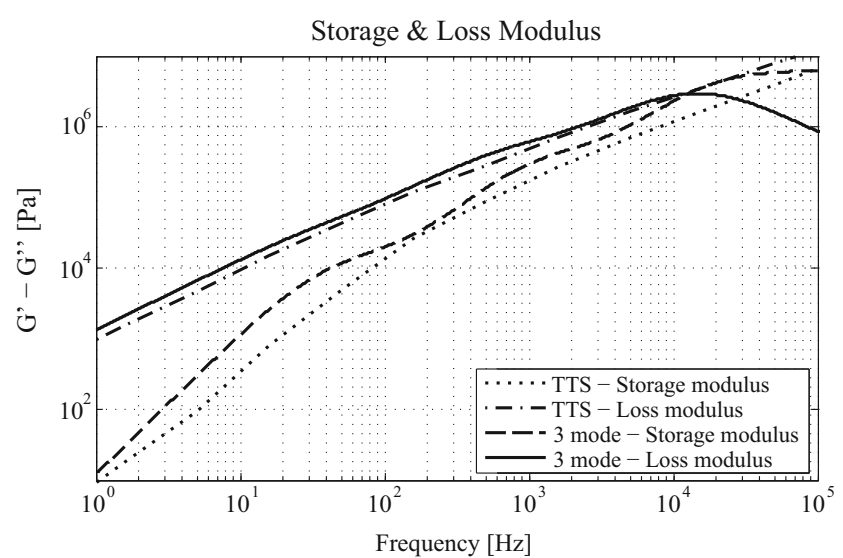

Fig. 12 Storage and loss moduli for a three-mode version of the fluid model and those obtained by time-temperature-superposition (TTS). The reference temperature is $20^{\circ} \mathrm{C}$

\section{Concluding remarks}

\section{Conclusion}

In this paper, a novel design of a sliding plate damper is presented that can be used to characterize LVE fluid behavior of high-viscosity fluids, i.e., it can be used as a (very cheap) rheometer. The drawbacks of standard sliding plate, damping devices, are taken cared off by the mechanical design: elastic elements are applied to enable a measurement stroke combined with stiff guidance in other directions. Linear viscoelastic fluid characterization of a high-viscosity fluid is performed in a frequency range from $10 \mathrm{~Hz}$ to $7 \mathrm{kHz}$. In this frequency range of roughly three decades, a three-mode Maxwell model accurately describes the LVE behavior. The fluid model obtained by the double-fin setup appeared to be more accurate than the fluid model obtained by the single-fin measurement, which was verified by the zero shear viscosity found of 209.7 Pa.s, which is close to the value as specified by the manufacturer. This indicates that a major part of the linear viscoelastic behavior of this fluid is captured in the frequency range that has been fitted. A second validation of the LVE behavior by TTS characterization shows that the fluid model as obtained is correctly up to $10 \mathrm{kHz}$. This frequency is high enough to obtain fluid models that can be used in practical damper designs for motion stages. The obtained LVE fluid models are applicable in the design procedure of dampers for future motion systems.

\section{Discussion}

The three-mode fluid model as described in this paper is intended for application in the design of industrial dampers for motion stages. This is demonstrated in
(Verbaan et al. to be submitted). Although this fluid model is applicable in practical designs, improvements can be made regarding the rheometer design, which improves the quality of the fluid model further. In order to derive the fluid model, the damper displacement is measured. The displacement graphs show, as function of frequency, successively, a region dominated by the stiffness, one dominated by the damping and one dominated by the mass. The damping behavior is dominant around the setup's resonance frequency. The single-fin measurement and the double-fin measurement show that a higher damping value leads to a larger damping-dominated frequency region and, therefore, to a more accurate fluid model. Figure 13 shows model-calculated behavior of respectively the undamped setup, the setup with the single-fin and double-fin mounted and an additional curve for a hypothetical setup with 10 fins. This curve shows that the damping becomes more dominant and, therefore, can be separated from the measurement with higher accuracy, especially at frequencies further from the resonance frequency: Fig. 13 shows that the damping behavior can be distinguished more accurately in the frequency regions $10-100 \mathrm{~Hz}$ and $3-10 \mathrm{kHz}$. This results in a higher quality of the fluid model, at least in these regions. Related to this observation is the question about the viscosity range of fluids that can be measured in this setup. Based on the previous statement about force-dominated frequency regions, this setup is not suitable for fluids with a zero shear viscosity lower than $100 \mathrm{~Pa} \cdot \mathrm{s}$. For lower viscosities, the damper design should be changed to obtain accurate fluid models. The geometrical factor of the damper has to be changed to increase the influence of the damping force. In addition, in this case, it might be possible to describe the fluid LVE behavior more accurately by applying more than three Maxwell modes.

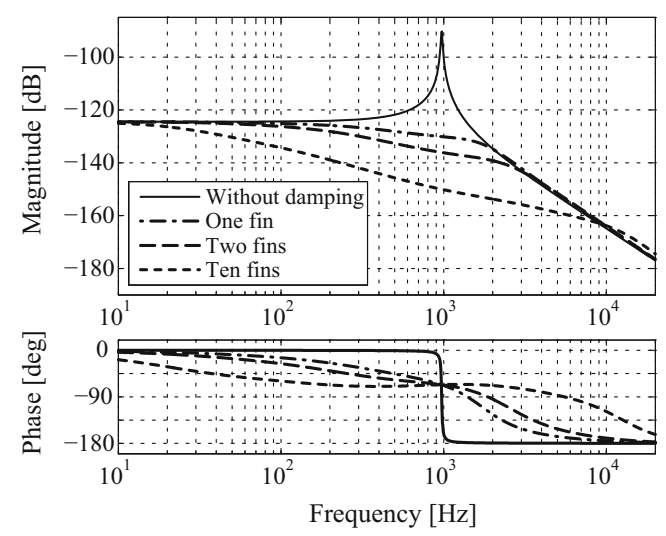

Fig. 13 Damper transfer functions based on the three-mode fluid model. The dotted line shows behavior for a damping mechanism with ten fins. This curve shows a larger frequency range that is dominated by the damping behavior 
Acknowledgments This project is supported by ASML Research Mechatronics (NL). We greatly appreciate the input of Dr.ir. J.P.M.B. Vermeulen, Dr.ir. M.M.J. van de Wal, and Dr.ir. S.H. van der Meulen.

In addition, we are grateful for the validation measurements which are kindly provided by the DSM Ahead Materials Science Center, Geleen, The Netherlands.

Open Access This article is distributed under the terms of the Creative Commons Attribution 4.0 International License (http:// creativecommons.org/licenses/by/4.0/), which permits unrestricted use, distribution, and reproduction in any medium, provided you give appropriate credit to the original author(s) and the source, provide a link to the Creative Commons license, and indicate if changes were made.

\section{References}

Bäck T (1996) Evolutionary algorithms in theory and practice. Oxford University Press, Oxford

Balas GJ, Doyle JC (1994) Control of lightly damped, flexible modes in the controller crossover region. AIAA J Guid Dyn Control 17(2): 370-377

Benzing DV, Russel WB (1981) The viscoelastic properties of ordered lattices: experiments. J Colloid Interface Sci 83:178

Bin, Yao, (2010), Advanced motion control; from classical PID to nonlinear adaptive robust control, IEEE 11th advanced motion control workshop, Nagaoka, 10.1109/AMC.2010.5464026

Blom C, Mellema J (1984) Torsion pendula with electromagnetic drive and detection system for measuring the complex shear modulus of liquids in the frequency range 80-2500 Hz. Rheol Acta 23:98-105

Bodden DS, Junkins JL (1985) Eigenvalue optimization algorithms for structure/controller design iterations. J Guid Control Dyn 8(6):697706

Book WJ (1993) Controlled motion in an elastic world. J Dyn Syst Meas Control 50:252-261

Crandall SH (1970) The role of damping in vibration theory. J Sound Vib 11(1):3-18

Crassous JJ, Régisser R, Ballauff M, Willenbacher N (2005) Characterization of the viscoelastic behavior of complex fluids using the piezoelastic axial vibrator. J Rheol 49(4):851-864

Diaz-Gomez, PA, Hougen DF (2007), Initial population for genetic algorithms: a metric approach

Ferry JD (1969) Viscoelastic properties of polymers. Wiley, New York

Fritz G, Pechhold W, Willenbacher N, Wagner NJ (2003) Characterizing complex fluids with high frequency rheology using torsional resonators at multiple frequencies. J Rheol 47(2):303-319 March/April

Gawronski WK (2004) Advanced structural dynamics and active control of structures. Springer_verlag, New York

Ho SL, Tomizuka M (1996) Robust motion controller design for highaccuracy positioning systems. IEEE Trans Ind Electron 43(1):48-55
Johnson CD (1995) Design of passive damping systems. J Vib Acoust 117(B):171-176

Konno A, Makino S, Kaneko M (1968) Measurements of the mechanical properties of a polymer solution. Jpn J Appl Phys 7:89

Macosko CW (1994) Rheology principles, measurements, and applications. Wiley, New York

Oakley JG, Giacomin AJ (1994) A sliding plate normal thrust rheometer for molten plastics. Polym Eng Sci 34(7):580-584

Olsen NB, Christensen T, Dyre JC (2001) Time-temperature superposition in viscous liquids. Phys Rev Lett 86(7):1271

Onishi K, Shibata M, Murakami T (1996) Motion control for advanced mechatronics. IEEE/ASME Trans Mechatron 1(1):56-67

Oosterbroek M, Waterman HA, Wiseall SS, Altena EG, Mellema J, Kip GAM (1980) Automatic apparatus, based upon a nickel-tube resonator, for measuring the complex shear modulus of liquids in the kHz range. Rheol Acta 19:497-506

Pechold W (1959) Eine method zur messing des komplexen schubmoduls im frequenzbereich 1-100 kHz. Acustica 9(1):39-48

Pedersen NL (2000) Maximization of eigenvalues using topology optimization. J Struct Multidiscip Optim 20(1):2-11

Rao SS, Rao SS (2009) Engineering optimization: theory and practice. Wiley, New York

Schrag JL (1977) Deviation of velocity gradients profiles from the 'gap loading' and 'surface loading' limits in dynamic simple shear experiments. Trans Soc Rhiol 21:399-413

Schrag JL, Johnson RM (1971) Application of the Birnboim multiple limped resonator principle to viscoelastic measurements of dilute macromolecular solutions. Rev Sci Instrum 42:244-232

Sittel K, Prince E, et al. (1954) Method for determining the viscoelastic properties of dilute polymer solutions at audiofrequencies. J Appl Phys 25:1312-1320

Skogestad S, Poslethwaite I (2005) Multivariable feedback controlanalysis and design, 2 edn. Wiley, New York

Soovere J, Drake ML, (1985), Aerospace structures technology damping design guide - Volume 1

Stokich TM, Radtke RR, White CC, Schrag JL (1994) An instrument for precise measurement of viscoelastic properties of low viscosity dilute macromolecular solutions at frequencies from 20 to $500 \mathrm{kHz}$. J Rheol 38:1195-1210

Swevers J, Pipeleers G, Diehl M, De Schutter J, (2010), Pushing motion control systems to their limits using convex optimization techniques, 11 th advanced motion control workshop, Nagaoka

Van de Wal M, De Jager B (2010) A review of methods for input/output selection. Automatica 37(4):487-510

Verbaan CAM, Rosielle PCJN, Steinbuch M, (2013), Broadband damping of non-rigid-body resonances of planar positioning stages by tuned mass dampers, Mechatronics (2014), 10.1016/j. mechatronics.2013.12.013

Verbaan, CAM, Rosielle, PCJN, Steinbuch, M, Suppression of flexible modes by applying robust mass dampers on a complex motion stage: open-loop damping optimization and experimental modal analysis. to be submitted 